\title{
Avaliação de dois programas de treinamento físico - concorrente e aeróbico - em mulheres obesas ou com sobrepeso portadoras da síndrome dos ovários policísticos
}

\author{
Evaluation of two physical training programs - concurrent and aerobic - in obese or overweight \\ women with polycystic ovary syndrome \\ Evaluación de dos programas de entrenamiento físico - concurrente y aeróbico - en mujeres obesas \\ o con sobrepeso con síndrome de ovario poliquístico
}

Recebido: 26/10/2021 | Revisado: 05/11/2021 | Aceito: 12/11/2021 | Publicado: 17/11/2021

Danielle Barbosa Santos
ORCID: https://orcid.org/0000-0001-9654-9847
Universidade Federal do Piauí, Brasil
E-mail: danielleprof79@ gmail.com
Laurilene Costa Silva
ORCID: https://orcid.org/0000-0002-6784-9178
Secretaria Estadual de Educação do Piauí, Brasil
E-mail: lauracslife@ @otmail.com
Denise Barbosa Santos
ORCID: https://orcid.org/0000-0002-8958-836X
Universidade Federal do Piauí, Brasil
E-mail: denisebarbosa@ @ufpi.edu.br
Fabio Rodrigues Trindade
ORCID: https://orcid.org/0000-0002-3667-5988
Universidade Federal do Piauí, Brasil
E-mail: fabiotrindade@ @ufpi.edu.br
Ione Maria Ribeiro Soares Lopes
ORCID: https://orcid.org/0000-0001-9008-3855
Universidade Federal do Piauí, Brasil
E-mail: ione.gin@ @otmail.com

\begin{abstract}
Resumo
Introdução: A Síndrome dos Ovários Policísticos (SOP) é um distúrbio endócrino, heterogêneo, cuja etiologia é desconhecida e com alta variação fenotípica .O tratamento de primeira linha para esta doença é o treinamento físico. Objetivo: Avaliar dois tipos de treinamentos físicos, concorrente e aeróbico, em mulheres obesas e com sobrepeso com SOP. Metodologia: Trata-se de um estudo clínico, analítico, prospectivo, controlado e randomizado, utilizando protocolos de treinamentos no "antes-depois", onde utilizou-se o treinamento aeróbico, que é a utilização do oxigênio como fonte de energia, e o treino concorrente, que é a utlização de treino de força e treino aeróbico. Foram selecionadas 30 mulheres, nas quais 14 , aproximadamente, $47 \%$ do total de pacientes participaram efetivamento do programa, com idade entre 18 e 40 anos com SOP obesas e/ou sobrepeso e posteriormente randomizadas para o Grupo I e Grupo II: a avaliação dos efeitos dos dois treinamentos, concorrente e aeróbico, sobre os parâmetros antropométricos, metabólicos e hormonais foi feita através de protocolos específicos para os treinos aeróbicos e concorrente.Foi utilizado para análise estatística o programa IBM SPSS versão 27.0. A distribuição normal dos dados foi realizado pelo teste de Shapiro-Wilk e para avaliar a mudança significativa da variável pós-intervenção, foi aplicado o teste t-student pareado. Resultados: Houve diferenças estatisticamente significante das variáveisestudadas nos parâmetros antropométricos no Treinamento Concorrente (TC) eTreinamento Aeróbico (TA) como peso, Índice de Massa Corporal (IMC), idademetabólica e gordura.O perfil da amostra demonstrou que dentre as selecionadas, a maioria $(42,86 \%)$ possuía ensino superior, $(57,14 \%)$ se autodeclararam negras e (100\%) eram sedentárias.Comparando-se o TA e TC relativo aos parâmetros antropométricos, os grupos apresentaram homogeneidade, com diferença somente na coxa $\mathrm{D}(\mathrm{P}=0,038)$. Conclusão: Os treinamentos físicos concorrentes e aeróbicos realizados três vezes por semana durante três meses reduzem de forma significativa vários parâmetros como peso, IMC, gorduras e parâmetros metabólicos. A eficácia é similar nos parâmetros antropométricos dos dois treinos físicos, concorrente e aeróbico.
\end{abstract}

Palavras-chave: Síndrome dos ovários polícisticos; Treinamento aeróbico; Treinamento concorrente.

\section{Abstract}

Introduction: Polycystic Ovary Syndrome (PCOS) is an endocrine disorder, heterogeneous, whose etiology is unknown and with high phenotypic variation. The first-line treatment for this disease is physical training. Objective: 
To evaluate two types of physical training, concurrent and aerobic, in obese and overweight women with PCOS. Methodology: This is a clinical, analytical, prospective, controlled and randomized study, using training protocols in the "before-after", where aerobic training was used, which is the use of oxygen as an energy source, and the concurrent training, which is the use of strength training and aerobic training. Thirty women were selected, of which 14, approximately $47 \%$ of the total number of patients participated in the program, aged between 18 and 40 years with obese and/or overweight PCOS, and subsequently randomized to Group I and Group II: the evaluation of the effects of the two training sessions, concurrent and aerobic, on anthropometric, metabolic and hormonal parameters was made through specific protocols for aerobic and concurrent training. The IBM SPSS version 27.0 program was used for statistical analysis. The normal distribution of data was performed using the Shapiro-Wilk test, and to assess the significant change in the post-intervention variable, the paired t-student test was applied. Results: There were statistically significant differences in the variables studied in the anthropometric parameters in Concurrent Training (TC) and Aerobic Training (AT) such as weight, Body Mass Index (BMI), metabolic age and fat. the majority (42.86\%) had higher education, (57.14\%) declared themselves black and (100\%) were sedentary. Comparing the AT and CT relative to anthropometric parameters, the groups were homogeneous, with difference only in thigh $\mathrm{D}(\mathrm{P}=0.038)$. Conclusion: Competitive and aerobic physical training performed three times a week for three months significantly reduce several parameters such as weight, BMI, fat and metabolic parameters. The effectiveness is similar in the anthropometric parameters of both physical training, concurrent and aerobic.

Keywords: Polycystic ovary syndrome; Aerobic training; Concurrent training.

\section{Resumen}

Introducción: El síndrome de ovario poliquístico (SOP) es un trastorno endocrino, heterogéneo, de etiología desconocida y con alta variación fenotípica, cuyo tratamiento de primera línea es el entrenamiento físico. Objetivo: Evaluar dos tipos de entrenamiento físico, concurrente y aeróbico, en mujeres obesas y con sobrepeso con SOP. Metodología: Se trata de un estudio clínico, analítico, prospectivo, controlado y aleatorizado, utilizando protocolos de entrenamiento en el "antes-después", donde se utilizó el entrenamiento aeróbico, que es el uso de oxígeno como fuente de energía, y el entrenamiento concurrente, que es el uso de entrenamiento de fuerza y entrenamiento aeróbico. Se seleccionaron 30 mujeres, de las cuales 14, aproximadamente el $47 \%$ del total de pacientes participaron en el programa, con edades comprendidas entre 18 y 40 años con SOP obesas y / o con sobrepeso, y posteriormente aleatorizadas al Grupo I y Grupo II: la evaluación de Los efectos de las dos sesiones de entrenamiento, concurrente y aeróbico, sobre los parámetros antropométricos, metabólicos y hormonales se realizaron mediante protocolos específicos para el entrenamiento aeróbico y concurrente. Para el análisis estadístico se utilizó el programa IBM SPSS versión 27.0. La distribución normal de los datos se realizó mediante la prueba de Shapiro-Wilk, y para evaluar el cambio significativo en la variable posintervención se aplicó la prueba t-student pareada. Resultados: Hubo diferencias estadísticamente significativas en las variables estudiadas en los parámetros antropométricos en Entrenamiento Concurrente (TC) y Entrenamiento Aeróbico (AT) como peso, Índice de Masa Corporal (IMC), edad metabólica y grasa. la educación superior, $(57,14 \%)$ se declararon negros y (100\%) sedentarios, comparando la TA y la TC con los parámetros antropométricos, los grupos fueron homogéneos, con diferencia solo en el muslo $\mathrm{D}(\mathrm{P}=$ 0,038). Conclusión: El entrenamiento físico competitivo y aeróbico realizado tres veces por semana durante tres meses reduce significativamente varios parámetros como el peso, el IMC, la grasa y los parámetros metabólicos. La efectividad es similar en los parámetros antropométricos tanto del entrenamiento físico, concurrente como aeróbico.

Palabras clave: Síndrome de ovario poliquístico; Entrenamiento aeróbico; Entrenamiento concurrente.

\section{Introdução}

A Síndrome dos Ovários Policísticos (SOP) é um distúrbio endócrino, heterogêneo, cuja etiologia é desconhecida e com alta variação fenotípica (Fauser et al., 2012).

Trata-se de uma doença que acomete de 5 - 10\% das mulheres em idade reprodutiva e frequentemente está associada a diversas alterações metabólicascomo Hipertensão Arterial Sistêmica (HAS) e Diabetes Tipo II (DT2) (Setji; Brown, 2014). De acordo com a Organização Mundial de Saúde (OMS), estima-se que, aproximadamente, 116 milhões de mulheres sejam afetadas por esse distúrbio em todo o mundo (Bharati et al., 2017).

Sendo assim, foram realizados vários consensos para estabelecer critérios para odiagnóstico da SOP, como o do National Institutes of Health (NIH), o de Rotterdam e o da Sociedade de hiperandrogênio e Síndrome dos Ovários Policísticos (AES- PCOS) (Azziz el al., 2006), contudo o mais utilizado é o Consenso de Rotterdam (2004), que estabelece a presença de dois dos três critérios: ovários policísticos ao ultrassom pélvico/transvaginal de 12 ou mais folículos periféricos e/ou volume ovariano superior a $10 \mathrm{~cm}^{3}$, oligo/anovulação (distúrbios menstruais) e por sinais clínicos e/ou laboratoriais de hiperandrogenismo. O diagnóstico de SOP só pode ser firmado após a exclusão de outras causas de hiperandrogenismo, como 
disfunções tireoidianas, hiperprolactinemias, hiperplasia adrenal congênita de manifestação tardia e tumores secretores de androgênios (Bulsara et al., 2021).

Para tanto, a SOP está associada à obesidade, dislipidemias, hipertensão e hiperinsulinemia. A hiperandrogenia e a hiperinsulinemia são as duas maiores características da SOP (Forslund et al., 2019).

Além de tudo, o maior risco cardiovascular em mulheres com esta patologia estárelacionado com a resistência à insulina, tolerância a glicose, dislipidemias, diabetes tipo 2, hipertensão e aterosclerose. A prevalência de síndrome metabólica é duas a quatro vezes maior nessa população (Kim, Choi, 2013; Sattar, 2006; Sattar, 2011). Além disso, é uma das maiores causadoras de infertilidade em mulheres em idade reprodutiva (Ehrmann, 2005; Tasali, 2008; Baranova,2011; Barnard et al., 2007; Delitala, 2017; Kandaraki, 2009).

Estas disfunções metabólicas ocasionadas pela SOP levam a ocorrência da obesidade agravando os riscos cardiometabólicos (Barber et al., 2007; Teede et al., 2013; Wild et al., 2000).

Sendo assim, de acordo com Barber et al., (2006) e Legro (2000), a maioria das mulheres com SOP (38\%-88\%) estão com sobrepeso ou obesas, sendo a obesidade central mais prevalente em mulheres com a doença, comparadas as que não possuem a síndrome (Hudecova et al., 2011; Sidhwani et al., 2011; Lim et al., 2012; Delitala et al., 2017).

Além das alterações metabólicas apresentadas, a SOP tem um impacto profundamente negativo na qualidade de vida das mulheres, diretamente na saúde mental e psicológica, com acometimento de quadros depressivos ou transtornos afetivos (Drosdzol, 2007; Teede et al., 2010).

Sendo assim, dentre as opções de tratamentos, tem-se o medicamentoso, incluindo hormônios, drogas antidiabéticas, diuréticos e o tratamento não-medicamentoso, que relaciona as terapias alternativas, como a yoga, até práticas de exercícios físicos (Bulsara et al., 2021).

Além disso, a mudança no estilo de vida é o tratamento não-farmacológico de primeira linha para a melhora na qualidade de vida (Hutchison et al., 2011).

O estilo de vida saudável, incluindo dieta nutritiva e equilibrada, ioga e exercícios físicos são considerados eficazes em mulheres com SOP, além disso, o exercício regular aumenta a qualidade de vida dessas mulheres e contribui para diminuir os níveis de insulina e andrógeno livre, o que pode levar à restauração do eixo hipotálamo-pituitário-gonadal da ovulação (Sharma; Srivastava, 2020).

Outrora, o tratamento não-medicamentoso era focado na normalização da anovulação e na redução dos parâmetros da síndrome metabólica. Sendo assim, aplicar programas de exercícios físicos, incluindo treinamento de força e aeróbico para perda de peso, podem ser suficientes para normalizar a função ovariana e, consequentemente, possibilitar a ocorrência da ovulação (Conte et al., 2015). Além disso, pode ser associado à melhoria na qualidade de vida relacionada àsaúde $\mathrm{e}$ outras complicações psicológicas em mulheres com SOP (Sharma; Srivastava, 2020).

Dentre os tipos de treinamentos físicos, encontra-se o Treinamento Aeróbico (TA) que tem como benefício reduzir a glicemia e adiposidade visceral além de melhorara qualidade de vida (Costa et al., 2018; Ribeiro et al., 2019). Estudos tem mostrado que o treinamento físico de força também pode melhorar os níveis hormonais de mulheres com SOP (Kogure et al., 2015), além de melhorar a resistência à insulina, metabolismo da glicose e taxa metabólica basal, redução da composição corporal, melhorando a sensibilidade à insulina em pacientes com diabetes tipo 2, promovendo também o aumento de força e massa magra (Ribeiro et al., 2019; Turan, 2015).

Nesse sentido, a literatura demonstra o impacto positivo dos exercícios físicos no tratamento das mulheres com SOP, principalmente nas inférteis, mas existe a dúvida se um tipo de treinamento físico teria melhor resultado do que outro, o que motivou a realização desse estudo.

Avaliar os efeitos de dois programas de treinamentos físicos: concorrente e aeróbico, na composição corporal, 
parâmetros metabólicos e endócrinos em mulheres com a Síndrome dos Ovários Policísticos (SOP) obesas ou com sobrepeso, atendidas no Hospital Universitário (HU-UFPI) em Teresina-PI.

O estudo teve como objetivos Específicos:

- Verificar os efeitos de dois treinamentos físicos (concorrente e aeróbico) através das medidas antropométricas de mulheres obesas ou com sobrepeso portadora da SOP, antes e após 12 semanas de cada treinamento físico;

- Verificar os efeitos de um treinamento concorrente e de outro aeróbico através da análise dos parâmetros metabólicos (colesterol total, glicemia de jejum, LDL, HDL, TG, HOMA-IR) em mulheres obesas ou com sobrepeso portadorasda SOP, antes e após 12 semanas de cada treinamento físico;

- Verificar os efeitos de dois treinamentos físicos: concorrente e aeróbico nos parâmetros hormonais (FSH, TSH e T4 livre, LH, prolactina, testosterona total e livre, androstenediona, insulina basal e 17 - Hidroxi-progesterona) em mulheres com SOP obesas ou com sobrepeso, antes e após 12 semanas de cada treinamento.

- Comparar os efeitos de um treinamento concorrente com um outro aeróbico através das medidas antropométricas de mulheres com SOP, obesas ou com sobrepeso, antes e após 12 semanas dos treinamentos físicos

\section{Metodologia}

\subsection{Caracterização do estudo e protocolo experimental}

Trata-se de um estudo clínico, analítico, prospectivo e randomizado, realizado no período de maio de 2019 a janeiro de 2021, em uma academia localizada na Associação dos Docentes da Universidade Federal do Piauí (ADUFPI) no bairro Ininga. A convocação de voluntárias participantes ocorreu nos meses de março de 2019 a outubro de 2020, no setor Saúde da mulher do Hospital Universitário HU- UFPI, na cidade de Teresina-PI, durante a consulta médica de mulheres com SOP que estavam em atendimento na instituição.

O local do estudo aparece no cenário estadual como instituição universitária e referência em tratamentos ginecológicos.

\subsection{População e seleção da amostra}

A população do estudo consistiu de 30 mulheres em idade reprodutiva, com diagnóstico de SOP que realizaram acompanhamento no ambulatório do HU/UFPI, Setor Saúde da mulher, no âmbito do Sistema Único de Saúde.

\subsection{Critérios de inclusão}

I. Mulher com idade de 18 a 40 anos com diagnóstico da SOP através dos critérios de Rotterdam: hiperandrogenismo clínico e/ou bioquímico, oligo ou anovulação e aspecto policístico dos ovários à ultrassonografia (12 ou mais folículos com diâmetro de 2 a 9mm e/ou aumento do volume ovariano $>10 \mathrm{~cm}^{3}$ ), afastando-se as outras causas que cursem com a mesmas características.

II. Ser obesa ou com sobrepeso, com Índice de Massa Corporal (IMC) $\geq 30 \mathrm{~kg} / \mathrm{m}^{2}$ para as obesas e IMC entre $25 \mathrm{~kg} / \mathrm{m}^{2}$ e $29,9 \mathrm{~kg} / \mathrm{m}^{2}$ para as que estão com sobrepeso.

III. Que não estejam em uso de medicamentos hormonais nos três meses que antecederam o estudo.

IV. Estar disposta a preencher adequadamente o termo de consentimento livre e esclarecido e ter disponibilidade para seguir as orientações que seriam repassadas antes, durante e após o protocolo de treinamento físico.

As mulheres pré-selecionadas foram avaliadas no Setor Saúde da Mulher do HU-UFPI, com a ginecologista 
responsável para a realização de exames prévios comprovação do diagnóstico da SOP.

O IMC foi a medida de referência utilizada para a formação dos grupos, considerando peso normal (de 18,5 a 24,9 $\left.\mathrm{kg} / \mathrm{m}^{2}\right)$, sobrepeso $\left(\geq 25 \mathrm{e}<30 \mathrm{~kg} / \mathrm{m}^{2}\right)$ e obesidade $(\geq 30 \mathrm{~kg} / \mathrm{m} 2)$.

\subsection{Critérios de exclusão}

I. Mulheres gestantes, lactantes e no período puerperal (menor ou igual a 12 semanas);

II. Uso de corticóides, medicamentos hormonais, medicações anti-androgênicas nos últimos 3 meses que antecederam o início do estudo;

III. Portadoras de doenças autoimunes, de endocrinopatias associadas à anovulação e hiperandrogenismo (hiperprolactinemia, hiperplasia congênita da suprarrenal nas formas clássica e não clássica, tireoidopatias, tumores produtores de androgênio e síndrome/doença de Cushing) ou doenças autoimunes;

IV. Mulheres que descontinuaram o treinamento;

V. Não aceitaram a assinar o Termo de Consentimento Livre e Esclarecido (TCLE).

Foram selecionadas inicialmente 30 mulheres que preencheram os critérios de inclusão para participarem do estudo e sorteadas para a inclusão nos 02 grupos, sendo o Grupo 1 (15 mulheres) - treinamento concorrente e Grupo 2 (15 mulheres)

- treinamento aeróbico.

A amostragem foi não probabilística, do tipo intencional, a partir de demanda espontânea das pacientes no setor e coletada no período do estudo.

Foram excluídas 16 mulheres, por não comparecerem assiduamente aostreinos (5), desistirem do programa devido às dificuldades de acesso ao local (8) e por não apresentarem os exames completos antes e depoisdo diagnóstico da SOP (3).

\subsection{Execução do Estudo}

Após a aprovação do projeto pelo CEP/UFPI, as participantes foram convocadas e distribuídas aleatoriamente em três dias da semana: segunda-feira, quarta-feira e sexta-feira, no horário de 18 às 19h, na academia dos Docentes da Universidade Federal do Piauí (ADUFPI) disponibilizada para a execução dos treinos. Dois estagiários foram selecionados para auxiliar na execução do protocolo detreinamento na academia, sob supervisão da profissional de educação física responsável.

Previamente, foi realizada avaliação física com métodos antropométricos através do profissional de educação física capacitado para a mensuração das medidas antropométricas. Segundo Da Costa (1999), o método antropométrico é a ciência que estuda e avalia as proporções do corpo humano e inclui as medidas: peso, estatura, circunferências corporais e espessura de dobras cutâneas, também avalia índices de doenças como o IMC e relação cintura-quadril (RCQ) (Rezende, 2007).

Após a avaliação física, foi executado o protocolo pela profissional responsável do treinamento especificado para cada grupo. Não foi realizado qualquer orientação dietética, sendo orientado somente a manutenção da alimentação cotidiana durante o período de treinamento.

As mulheres foram elegíveis independente de raça, paridade, condição financeira e escolaridade, sendo respeitados os critérios de inclusão e exclusão.

\subsection{Instrumento de coleta de dados e protocolo experimental}

As informações de interesse da pesquisa foram obtidas com todasas participantes durante consulta médica, por meio de anamnese, exame físico completo, avaliação de exames complementares e acesso ao prontuário eletrônico do HU-UFPI.

Para aconfirmação diagnóstica da SOP foram realizados exames de ultrassonografia pélvica ou transvaginal e os bioquimicos, onde foram observados os critérios do Consenso de Rotterdam, que considera SOP a presença de pelo menos 
duas das três características apresentadas pela síndrome: oligomenorréia ou anovulação, sinais clínicos (hirsutismo e/ou acne) e/ou bioquímicos de hiperandrogenismo, a identificação de ovários policísticos por meio da ultrassonografia, com a presença de 12 ou mais folículos, medindo de 2 a 9 milímetros de diâmetro e/ou volume ovariano acima de $10 \mathrm{~cm}^{3}$, após afastar outras causas de hiperandrogenismo (hipotireoidismo, hiperprolactinemia, hiperplasia adrenal congênita de manifestação tardia ou tumores adrenais) (Consensus- Pcos-Rotterdam, 2004).

No exame físico e ginecológico, foram realçados o grau de hirsutismo classificado pela tabela proposta por Ferriman e Gallway (1961), modificada por Hatch et al. (1981), que graduaram os pelos de 0 a 4 em 9 regiões do corpo e consideraram hirsutismo quando o total for $>8$ (Figura 1) (Teede et al., 2018).

Figura 1 - Escala de Ferriman-Gallwey.

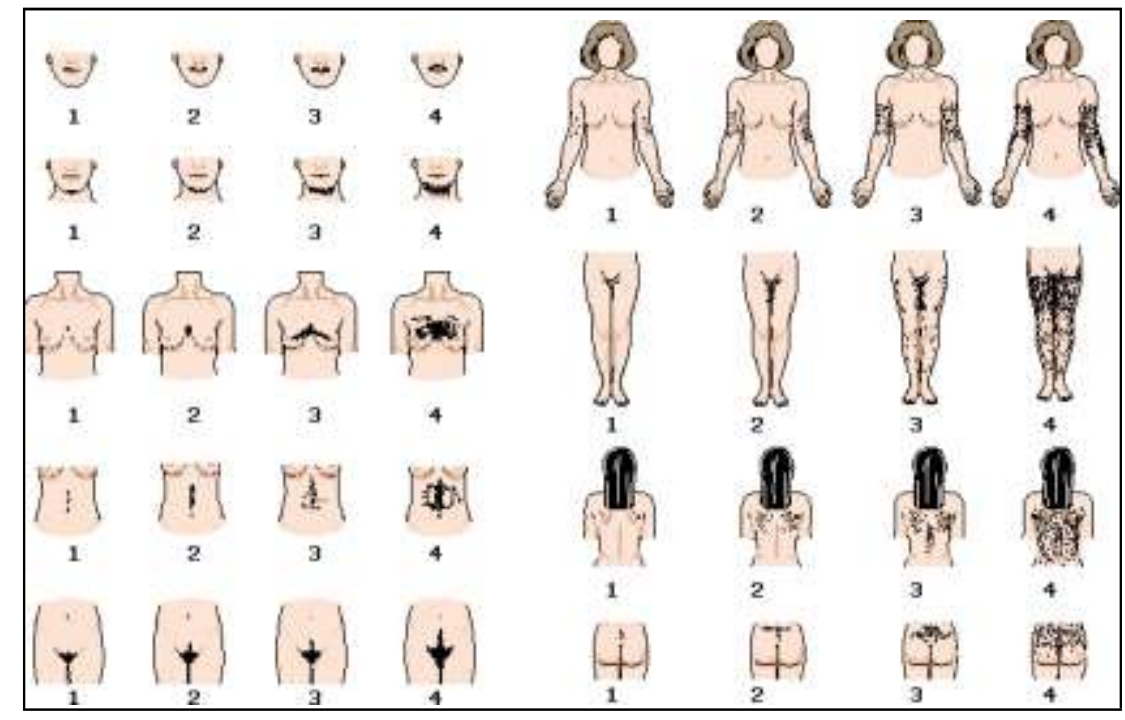

Fonte: Autores.

$\mathrm{Na}$ coleta de dados, inicialmente, foi utilizado um formulário pré-estruturado em que constam dados sociodemográficos (idade, etnia autodeclarada), estado marital, escolaridade, dados antropométricos (IMC, circunferência abdominal e quadril) estilo de vida (etilismo, tabagismo, prática de atividade física), aspectos clínicos (doenças pessoais e familiares pregressas, ciclo menstrual presença de hirsutismo), bioquímicos: glicemia de jejum, teste oral de tolerância à glicose em jejum e 2 horas após sobrecarga (TOTG 75g), Low Density Lipoproteins (LDL), High Density Lipoproteins (HDL), triglicerídeos, hormônio folículo estimulante (FSH), hormônio estimulante da tireóide (TSH), tiroxina livre (T4 livre), hormônio luteinizante (LH), prolactina, testosteronas total e livre, androstenediona, insulinemia basal, 17-hidroxiprogesterona (17-OH-P) e HOMA-IR.

Após a seleção e inclusão no estudo, as participantes realizaram uma avaliação física realizada pela pesquisadora, incluindo a mensuração da massa corporal, IMC, percentual de gordura corporal, percentual de massa muscular, metabolismo basal, percentual de gordura visceral e idade metabólica através do aparelho de bioimpedância elétrica bipolar Beurer BF-100. Foram incluídas também medidas de circunferência do braço, antebraço, peitoral, cintura, abdômen, quadril, coxas e panturrilhas, utilizando a fita antropométrica da marca Sanny. A medida da circunferência da cintura, foi feita entre a última costela e a crista íliaca.

O IMC foi a medida de referência utilizada para a formação dos grupos, considerando peso normal (de 18,5 a 24.9 $\left.\mathrm{kg} / \mathrm{m}^{2}\right)$, sobrepeso $\left(\geq 25 \mathrm{e}<30 \mathrm{~kg} / \mathrm{m}^{2}\right)$ e obesidade $\left(\geq 30 \mathrm{~kg} / \mathrm{m}^{2}\right)$ (Abeso, 2016).

Para os protocolos de treinamentos físicos foram utilizadas 6 sessões de adaptação, sendo que para o treinamento concorrente foi utilizado em cada sessão 2 séries de 15 repetições com a menor carga possível, com frequência de 3 vezes na 
semana, com duração de 60 minutos, durante 2 semanas.

Em relação ao treino aeróbico foi utilizado sessão com FC máxima de $60 \%$ em esteira ergométrica da marca Moviment. Para a realização do treinamento concorrente (GRUPO 1) foi executado, inicialmente o teste de 1 Repetição Máxima (RM), onde foi utilizado o exercício supino reto com barra, com tempo estimado de 3-5 minutos de atividade envolvendo o grupo muscular peitoral, para registrar a carga máxima alcançada (Materko; Neves; Santos, 2007). O protocolo do treinamento concorrente (Grupo 1) constou de 3 microciclos, sendo cada microciclo constituído de 4 semanas. A prescrição do treinamento foi a mesma para todas as participantes, com cargas individualizadas. Inicialmente, a cada sessão foi realizado aquecimento de 5 minutos em bicicleta ergométrica ou esteira, e posteriormente, a sessão de exercícios combinados, alternando membros superiores e inferiores, com duração de 30 minutos. No primeiro microciclo foi realizado 3 séries de 15 repetições, com carga submáxima, no segundo microciclo 3 séries de 12 repetições aumentando-se a carga a cada semana, e no último microciclo com 10 repetições aumentando-se a carga a cada semana.

Em todas as séries foram respeitados 40 segundos de recuperação. Após o término do treino resistido devia-se realizar, imediatamente, o treinamento aeróbio com duração de 30 minutos (Rossi, 2016), utilizando-se esteira ergométrica, bicicleta ou elíptico.

A intensidade do treinamento resistido foi determinada pelo Esforço Nominal Percebido (EPR), onde a carga de trabalho foi ajustada de acordo com a percepção individual do esforço (Figura 2) (Borg, 1982).

Figura 2 - Escala de percepção subjetiva de esforço (PSE). Teresina, Piauí, Brasil,2021. (Fonte: Adaptado de Borg, 1982).

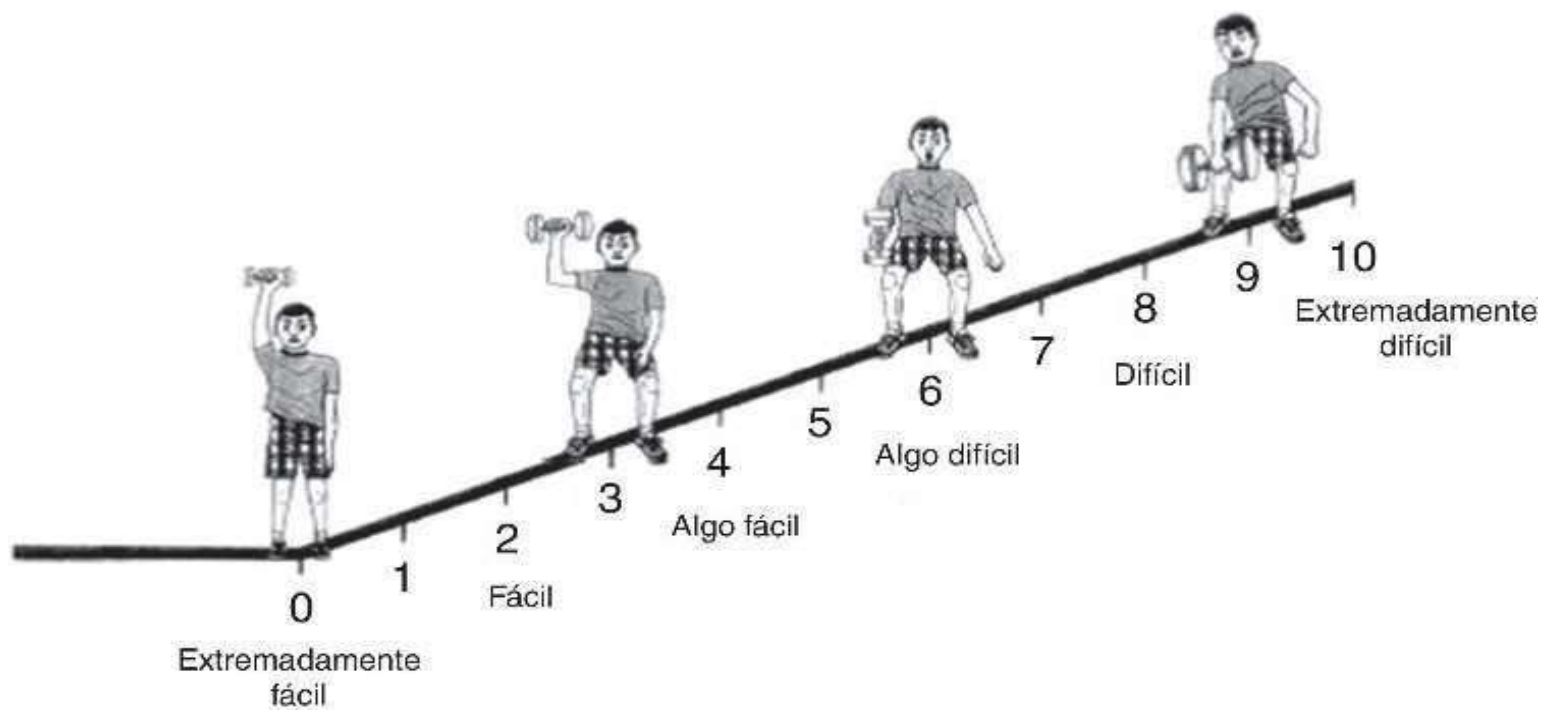

Fonte: Autores.

Para o treinamento resistido foram utilizados os aparelhos: peitoral voador, cadeira extensora, supino reto, legpress $180^{\circ}$, flexora horizontal, remada sentada, panturrilha sentada, bíceps braquial, tríceps pulley e por fim, 3 séries de 20 repetições de exercícios abdominais.

Para o protocolo do treinamento aeróbico (Grupo 2), a duração foi de 60 minutos, constituído pela esteira, elíptico e bicicleta ergométrica, inicialmente com FC máximo de 60 a 75\%, utilizando-se de frequencímetro de pulso POLAR 810 e, no decorrer das semanas foi-se aumentando a intensidade de acordo com a capacidade de aptidão física de cada participante.

Durante toda a execução do programa, a pesquisadora profissional de educação física esteve presente orientando as participantes. Após o término das 12 semanas, as pacientes foram submetidas a nova avaliação física e laboratorial para efeito 
dos resultados.

\subsection{Procedimentos para análise dos dados}

A análise estatística foi realizada para cada tipo de intervenção (Concorrente e Aeróbico) utilizando o programa IBM SPSS Statistics versão 27.0. A distribuição normal dos dados foi realizada pelo teste de Shapiro-Wilk, devido ao número de observações serem < 30. Para avaliar a existência de mudança significativa da variável após intervenção, foi aplicado o teste $t$ Student pareado, quando apresentavam normalidade de dados. Quando não havia essa condição, foi utilizado o teste de Wilcoxon e considerou-se o nível de significância a 5\%.

\subsection{Aspectos éticos}

Essa pesquisa cumpriu todas as diretrizes das Resoluções 466/2012 e 510/2016 do Conselho Nacional de Saúde e foi aprovada pelo Comitê de Ética em Pesquisa da Universidade Federal do Piauí, com o parecer consubstanciado de nº 3.949.315. As participantes assinaram o Termo de Consentimento Livre e Esclarecido (TCLE) e receberam uma via do mesmo.

\section{Resultados}

\subsection{Perfil da amostra}

Foram avaliadas e selecionadas 30 mulheres, destas, excluiu-se 16 participantes ao longo do estudo. Assim, a amostra final foi representada por 14 participantes, sendo 7 participantes do Grupo I e 7 participantes do Grupo II.

A Tabela 1 dispõe o perfil das mulheres que participaram do estudo. A maioriatinha idade igual ou superior aos 30 anos $(85,71 \%)$, casadas $(78,57 \%)$, se autodeclaram negras $(57,14 \%), 42,86 \%$ possuíam ensino superior completo, seguido de ensino médio completo $(35,71 \%)$. Todas as voluntárias eramsedentárias. 
Tabela 1 - Perfil sociodemográfico das participantes do estudo. Teresina, Piauí,Brasil, 2020.

\begin{tabular}{|c|c|c|}
\hline Características & $\mathrm{N}$ & $\%$ \\
\hline \multicolumn{3}{|l|}{ Idade } \\
\hline$<30$ & 2 & 14,29 \\
\hline$\geq 30$ & 12 & 85,71 \\
\hline \multicolumn{3}{|l|}{ Escolaridade } \\
\hline Ensino médio incompleto & 1 & 7,14 \\
\hline Ensino médio completo & 5 & 35,71 \\
\hline Ensino superior incompleto & 2 & 14,29 \\
\hline Ensino superior completo & 6 & 42,86 \\
\hline \multicolumn{3}{|l|}{ Cor de pele } \\
\hline Branca & 6 & 42,86 \\
\hline Negra & 8 & 57,14 \\
\hline \multicolumn{3}{|l|}{ Estado civil } \\
\hline Casada & 11 & 78,57 \\
\hline Solteira & 3 & 21,43 \\
\hline \multicolumn{3}{|l|}{ Sedentarismo } \\
\hline Sim & 14 & 100,00 \\
\hline Não & 0 & 0,00 \\
\hline
\end{tabular}

Fonte: Autores.

\subsection{Análise dos efeitos do treinamento concorrente (TC) sobre os parâmetrosantropométricos}

$\mathrm{Na}$ Tabela 2 tem-se a diferença pré e pós TC quanto aos dados antropométricos. É possível observar que houve redução significativa em vários parâmetros como peso, IMC e gordura corporal ( $\mathrm{p}=0,028)$, aumento do músculo ( $\mathrm{p}=0,018)$, redução da idade metabólica $(p=0,027)$ e diminuição da circunferência do antebraço esquerdo $(p=0,024)$. A cintura e quadril também apresentaram reduções relevantes $(\mathrm{p}=0,028)$. Outras medidas apresentaram diferenças, contudo não foram estatisticamente significantes. 
Tabela 2 - Comparação dos dados antropométricos pré e pós treinamento concorrente em mulheres com SOP obesas ou sobrepeso. Teresina, Piauí, Brasil, 2020.

\begin{tabular}{|c|c|c|c|c|c|c|}
\hline \multirow{2}{*}{ Variável } & \multirow{2}{*}{$\begin{array}{c}\text { Antes } \\
\text { Mediana }\end{array}$} & \multicolumn{3}{|c|}{ Depois } & \multirow{2}{*}{$\begin{array}{c}\text { Variação } \\
(\%)\end{array}$} & \multirow{2}{*}{ valor $p$} \\
\hline & & $\mathrm{IQ}^{*}$ & Mediana & $\mathrm{IQ}^{*}$ & & \\
\hline Peso & 79 & 10 & 74,6 & 10,1 & $-5,6$ & $0,028 \mathrm{a}$ \\
\hline IMC & 28,1 & 5,7 & 28 & 3,9 & $-0,4$ & $0,028 \mathrm{a}$ \\
\hline Gordura & 45 & 5,5 & 42 & 4 & $-6,7$ & $0,028 \mathrm{a}$ \\
\hline Músculo & 23 & 1,9 & 24 & 1,9 & 4,3 & $0,018 \mathrm{a}$ \\
\hline MetBas & 1490 & 120 & 1425 & 116 & $-4,4$ & $0,063 \mathrm{a}$ \\
\hline Idade Met & 56 & 17 & 51 & 17 & $-8,9$ & $0,027 \mathrm{a}$ \\
\hline Visceral & 8 & 3 & 7 & 2 & $-12,5$ & $0,063^{\mathrm{a}}$ \\
\hline Braço Direito & 33 & 4,1 & 33 & 4,1 & 0,0 & $1,000^{\mathrm{a}}$ \\
\hline Braço Esq & 32 & 3 & 32 & 3 & 0,0 & $1,000^{\mathrm{a}}$ \\
\hline Antebraço D & 26 & 2 & 26 & 1,5 & 0,0 & $0,157^{\mathrm{a}}$ \\
\hline Antebraço E & 25,5 & 2 & 25 & 1 & $-2,0$ & $\mathbf{0 , 0 2 4 ^ { a }}$ \\
\hline Peitoral & 97 & 6,5 & 95 & 3 & $-2,1$ & $0,206^{\mathrm{a}}$ \\
\hline Cintura & 85 & 13 & 82 & 16 & $-3,5$ & $0,028^{a}$ \\
\hline Abdominal & 90 & 12 & 87 & 11 & $-3,3$ & $0,034^{\mathrm{a}}$ \\
\hline Quadril & 110 & 8 & 104 & 10 & $-5,5$ & $0,028^{\mathrm{a}}$ \\
\hline Coxa D & 65 & 7,5 & 64 & 6 & $-1,5$ & $0,593^{\mathrm{a}}$ \\
\hline Coxa E & 64 & 3,5 & 63,5 & 31 & $-0,8$ & $0,276^{\mathrm{a}}$ \\
\hline Panturrilha D & 37,5 & 5 & 38 & 4 & 1,3 & $0,102^{\mathrm{a}}$ \\
\hline Panturrilha E & 37 & 3,5 & 38 & 3,5 & 2,7 & $0,157^{\mathrm{a}}$ \\
\hline
\end{tabular}

Fonte: Autores.

\subsection{Análise dos efeitos do treinamento aeróbico (TA) sobre os dadosantropométricos}

A Tabela 3 apresenta a diferença pré e pós TA quanto aos dados antropométricos. As maiores reduções foram quanto ao peso $(\mathrm{p}=0,000)$, IMC $(\mathrm{p}=0,018)$ e gordura $(\mathrm{p}=0,018)$. Ainda na tabela 04 é possível observar redução significativa no metabolismo basal $(\mathrm{p}=0,018)$, idade metabólica $(\mathrm{p}=0,018)$, visc. $(\mathrm{p}=0,015)$, antebraço $\mathrm{E}(\mathrm{p}=0,038)$, peitoral $(0,041)$, cintura $(0,017)$, abdômen $(\mathrm{p}=0,018)$, quadril $(\mathrm{p}=0,018)$. 
Tabela 3 - Comparação dos dados antropométricos pré e pós treinamentoaeróbico. Teresina, Piauí, Brasil, 2020.

\begin{tabular}{|c|c|c|c|c|c|c|}
\hline \multirow{2}{*}{ Variável } & \multicolumn{2}{|c|}{ Antes } & \multicolumn{2}{|c|}{ Depois } & \multirow{2}{*}{$\begin{array}{c}\text { Variação } \\
(\%)\end{array}$} & \multirow{2}{*}{ valor $\mathbf{p}$} \\
\hline & Mediana & $\mathbf{I Q}^{*}$ & Mediana & $\mathbf{I Q}^{*}$ & & \\
\hline Peso & $82,07^{* *}$ & $14,03^{* * *}$ & $75,61^{* *}$ & $12,96^{* * *}$ & $-7,9$ & $0,000^{b}$ \\
\hline IMC & 32 & 12,8 & 29,1 & 13,6 & $-9,1$ & $0,018^{\mathrm{a}}$ \\
\hline Gordura & 48 & 11,4 & 43,8 & 12,4 & $-8,8$ & $0,018^{a}$ \\
\hline Musculo & 22,6 & 2,1 & 24,4 & 3,4 & 8,0 & $0,270^{\mathrm{a}}$ \\
\hline MetBasal & 1470 & 210 & 1361 & 214 & $-7,4$ & $0,018^{a}$ \\
\hline Idade Met & 63 & 24 & 57 & 26 & $-9,5$ & $0,018^{a}$ \\
\hline Visceral & 9 & 5 & 8 & 4 & $-11,1$ & $0,015^{a}$ \\
\hline Braço D & $34,42^{* *}$ & $2,62^{* * * *}$ & $35,71^{* *}$ & $4,14^{* * * *}$ & 3,7 & $0,356^{\mathrm{b}}$ \\
\hline Braço E & 32,5 & 5 & 32,5 & 5 & 0,0 & $1,000^{\mathrm{a}}$ \\
\hline Antebraço D & 26 & 6,5 & 26 & 8 & 0,0 & $0,157^{\mathrm{a}}$ \\
\hline Antebraço E & $26,21^{* *}$ & $2^{* * *}$ & $25,71^{* *}$ & $2,36^{* * *}$ & $-1,9$ & $\mathbf{0 , 0 3 8} 8^{b}$ \\
\hline Peitoral & 101 & 6 & 100 & 6 & $-1,0$ & $0,041^{a}$ \\
\hline Cintura & 86,5 & 22 & 84 & 14,5 & $-2,9$ & $0,017^{\mathrm{a}}$ \\
\hline Abdominal & 100 & 27 & 92 & 20 & $-8,0$ & $0,018^{a}$ \\
\hline Quadril & 109,5 & 29 & 100 & 24 & $-8,7$ & $0,018^{a}$ \\
\hline Coxa direita & $63,79^{* *}$ & 5,43 & $63,86^{* *}$ & $5,3^{* * *}$ & 0,1 & $0,689^{b}$ \\
\hline $\begin{array}{c}\text { Coxa } \\
\text { esquerda }\end{array}$ & 59 & 7 & 59 & 7 & 0,0 & $1,000^{\mathrm{a}}$ \\
\hline Panturrilha D & $37,57^{* *}$ & $5,26^{* * * *}$ & $37,43^{* *}$ & $4,82^{* * *}$ & $-0,4$ & $0,569^{\mathrm{b}}$ \\
\hline Panturrilha E & $36,67^{* *}$ & $3,44^{* * *}$ & $36,86^{* *}$ & $3,72^{* * *}$ & 0,5 & $0,460^{\mathrm{b}}$ \\
\hline
\end{tabular}

Legenda: Antebraço D: antebraço direito, Antebraço E: antebraço esquerdo, Panturrilha D: panturrilha direita, Panturrilha E: panturrilha esquerda, Met basal: metabolismo basal, Idade Met: idade metabólica. "IQ: Intervalo Interquartílico; ${ }^{* *}$ Média; ${ }^{* * *}$ Desvio-Padrão. ${ }^{a}$ Teste de Wilcoxon. ${ }^{\text {b}}$ Teste t de Student pareado. Fonte: Autores.

\section{Análise dos efeitos do treinamento concorrente (TC) nos parâmetrosmetabólicos e hormonais}

Na Tabela 4, os dados demonstram a diferença pré e pós TC nos resultados de exames hormonais e metabólicos. Foram obtidas mudanças significativas em vários parâmetros como redução do TOTG-75g-2h (p=0,018), aumento da 17alfa- hidroxiprogesterona ( $\mathrm{p}=0,018)$ e redução acentuada dos triglicerídeos e colesterol $\mathrm{T}(\mathrm{p}=0,018)$. 
Tabela 4 - Comparação dos resultados dos parâmetros metabólicos e hormonais pré e pós treinamento concorrente em mulheres com SOP obesas ou sobrepeso. Teresina, Piauí, Brasil, 2020.

\begin{tabular}{ccccccc}
\hline Variável & $\begin{array}{c}\text { Antes } \\
\text { Mediana }\end{array}$ & IQ* & Mediana & Depois & $\begin{array}{c}\text { Variação } \\
(\mathbf{\%})\end{array}$ & valor p \\
\hline TOTG & 99 & 61 & 79 & 33 & $-20,2$ & $\mathbf{0 , 0 1 8}^{\mathbf{a}}$ \\
HDL & 52 & 24 & 49 & 4 & $-5,8$ & $0,933^{\mathrm{a}}$ \\
T4 & 1,02 & 0,19 & 1,05 & 0,27 & 2,9 & $0,612^{\mathrm{a}}$ \\
FSH & 5,17 & 4,16 & 4,46 & 3,64 & $-13,7$ & $0,499^{\mathrm{a}}$ \\
Test total & 40,1 & 21,7 & 33,6 & 22,8 & $-16,2$ & $0,271^{\mathrm{a}}$ \\
Test livre & 0,5 & 0,84 & 0,4 & 0,453 & $-20,0$ & $0,176^{\mathrm{a}}$ \\
17-OH-P & 110 & 208,2 & 125 & 317 & 13,6 & $\mathbf{0 , 0 1 8 ^ { \mathrm { a } }}$ \\
Insulina & 12 & 9,24 & 12,8 & 6,65 & 6,7 & $0,866^{\mathrm{a}}$ \\
HOMA-IR & $3,99^{* *}$ & $4,32^{* * *}$ & $4,67^{* *}$ & $6,26^{* * *}$ & 17,0 & $0,460^{\mathrm{b}}$ \\
LDL & 108 & 30 & 94 & 47 & $-13,0$ & $0,345^{\mathrm{a}}$ \\
Triglicerideos & 132 & 48 & 92 & 48 & $-30,3$ & $\mathbf{0 , 0 1 8 ^ { \mathbf { a } }}$ \\
TSH & 1,52 & 0,72 & 1,47 & 1 & $-3,3$ & $0,672^{\mathrm{a}}$ \\
LH & 10,1 & 9,39 & 8,19 & 5,98 & $-18,9$ & $0,176^{\mathrm{a}}$ \\
Prolactina & $16,41^{* *}$ & $12,28^{* * *}$ & $17,65^{* *}$ & $11,15^{* * *}$ & 7,6 & $0,218^{\mathrm{b}}$ \\
Glicemia & 77 & 12 & 80 & 25 & 3,9 & $1,000^{\mathrm{a}}$ \\
Andros & 1,7 & 1,57 & 2 & 1,87 & 17,6 & $0,397^{\mathrm{a}}$ \\
Colest T & 178 & 31 & 140 & 44 & $-21,3$ & $\mathbf{0 , 0 1 8}^{\mathbf{a}}$ \\
\hline
\end{tabular}

Legenda:TOTG: Teste Oral de Tolerância a Glicose; FSH: Hormônio Folículo estimulante; TSH: Hormônio Tireoestimulante. LH: Hormônio Luteinizante; 17-OH=P:;HDL:high-densitylipoprotein; LDL:low-densitylipoprotein;Colest T: colesterol total, Test total: testosterona total, Test livre: testosterona livre, Andros: androstenediona, 17-hidroxi: 17hidroxiprogesterona. ${ }^{*}$ Q: Intervalo Interquartílico; ${ }^{* *}$ Média; ${ }^{* * *}$ Desvio-Padrão. ${ }^{\text {T}}$ Teste de Wilcoxon. ${ }^{\text {b}}$ Teste $\mathrm{t}$ de Student pareado.

Fonte: Autores.

\subsection{Análise dos efeitos do treinamento aeróbico (TA) nos parâmetrosmetabólicos e hormonais}

Na Tabela 5 estão dispostos os resultados dos exames bioquímicos metabólicos e hormonais, antes e após o TA. Houve redução significativa em TOTG- 75-2h (p=0,042), HDL ( $\mathrm{p}=0,016)$, LDL ( $\mathrm{p}=0,042)$, glicemia ( $\mathrm{p}=0,028)$ e ColesterolTotal $(\mathrm{p}=0,028)$. Outros parâmetros não foram estatisticamente significantes. Os níveis de TOTG-75-2h e Colesterol Total tiveram maiores reduções no TC em relação ao TA e no TC foi possível ainda observar aumento acentuado da hidroxiprogesterona e redução dos triglicerídeos. 
Tabela 5 - Comparação dos resultados dos parâmetros metabólicos e hormonais pré e pós treinamento aeróbico em mulheres com SOP obesas ou sobrepeso. Teresina, Piauí, Brasil, 2020.

\begin{tabular}{|c|c|c|c|c|c|c|}
\hline \multirow[t]{2}{*}{ Variável } & \multicolumn{2}{|l|}{ Antes } & \multicolumn{2}{|c|}{ Depois } & \multirow{2}{*}{$\begin{array}{c}\text { Variação } \\
(\%)\end{array}$} & \multirow[t]{2}{*}{ valor $p$} \\
\hline & Mediana & $\mathrm{IQ}^{*}$ & Mediana & $\mathrm{IQ}^{*}$ & & \\
\hline TOTG & 105 & 68 & 102 & 30 & $-2,9$ & $0,042 \mathrm{a}$ \\
\hline HDL & 40,5 & 26 & 35,3 & 23 & $-12,8$ & $0,016 \mathrm{a}$ \\
\hline $\mathrm{T} 4$ & 0,91 & 0,25 & 1,02 & 0,22 & 12,1 & $0,061 \mathrm{a}$ \\
\hline FSH & 4,9 & 2,73 & 4,75 & 1,27 & $-3,1$ & $0,735 \mathrm{a}$ \\
\hline Test $\mathrm{T}$ & 40,8 & 57,2 & 40,7 & 32,1 & $-0,2$ & $0,236 \mathrm{a}$ \\
\hline Test L & 1,08 & 0,39 & 1,07 & 0,46 & $-0,9$ & $0,176 \mathrm{a}$ \\
\hline 17-OH-P & 104 & 189,54 & 179 & 128 & 72,1 & $0,176 a$ \\
\hline Insulina & $25,77 * *$ & $21,1 * * *$ & $28,64 * *$ & $26,78 * * *$ & 11,1 & $0,506 \mathrm{~b}$ \\
\hline HOMA-IR & 3,75 & 3,08 & 2,39 & 6,62 & $-36,3$ & $1,000 \mathrm{a}$ \\
\hline LDL & 118,4 & 14 & 93,6 & 47,9 & $-20,9$ & $0,042 \mathrm{a}$ \\
\hline Triglicerídeos & 165 & 149 & 153 & 111 & $-7,3$ & $0,932 \mathrm{a}$ \\
\hline TSH & 2,41 & 1,98 & 3,1 & 1,1 & 28,6 & $0,735 \mathrm{a}$ \\
\hline LH & 15,2 & 10,57 & 11,5 & 15,29 & $-24,3$ & $0,866 \mathrm{a}$ \\
\hline Prolactina & 17,7 & 12,2 & 18,9 & 10,31 & 6,8 & $1,000 \mathrm{a}$ \\
\hline Glicemia & 93 & 13 & 84 & 6 & $-9,7$ & $0,028^{a}$ \\
\hline Androst & 1,5 & 3,6 & 2,47 & 4 & 64,7 & $0,090^{\mathrm{a}}$ \\
\hline Colesterol T & 190 & 36 & 150 & 52 & $-21,1$ & $0,028^{a}$ \\
\hline
\end{tabular}

Legenda: Colesterol T: colesterol total, Test T: testosterona total, Test L: testosterona livre, Androst: androstenediona, 17-OH=P: 17- hidroxiprogesterona; HDL:high-densitylipoprotein; LDL:low- densitylipoprotein;. ${ }^{*} \mathrm{IQ}$ : Intervalo Interquartílico; ${ }^{* *}$ Média; ${ }^{* * *}$ Desvio-Padrão. ${ }^{\text {TTeste de Wilcoxon. }}{ }^{\text {bTeste }}$ t de Student pareado.

Fonte: Própria.

3.5 Análise comparativa entre os grupos de treinamento aeróbico (TA) etreinamento concorrente (TC) quanto aos parâmetros antropométricos

Na Tabela 6 têm-se a comparação quanto aos parâmetros antropométricos entre os grupos TA e TC no que tange os parâmetros antropométricos. Os grupos secomportaram de maneira homogênea, atingindo diferença estatística apenas na $\operatorname{coxa} \mathrm{D}(\mathrm{p}=0,038)$. 
Tabela 6 - Comparação dos resultados dos parâmetros antropométricos entre os grupos de treinos aeróbico e concorrente em mulheres com SOP obesas ou sobrepeso. Teresina, Piauí, Brasil, 2020.

\begin{tabular}{|c|c|c|c|c|c|c|c|}
\hline & \multicolumn{3}{|c|}{ GRUPOS } & \multirow{2}{*}{\multicolumn{3}{|c|}{ Treino concorrente }} & \multirow{3}{*}{ P-valor } \\
\hline & & reino aeróbico & & & & & \\
\hline & Média & Desvio padrão & Mediana & Média & Desvio padrão & Mediana & \\
\hline PESO & -3.6 & 2.7 & -3.1 & -3.7 & 2.7 & -3.6 & 0.954 \\
\hline IMC & -1.0 & 1.4 & -1.0 & -1.1 & 1.4 & -1.2 & 0.926 \\
\hline Gordura & -2.0 & 2.4 & -2.0 & -1.4 & 2.0 & -1.8 & 0.593 \\
\hline Músculo & 0.8 & 1.0 & 0.5 & 0.7 & 0.7 & 0.9 & 0.905 \\
\hline MET BASAL & -91.9 & 86.8 & -54.0 & -29.7 & 31.9 & -38.0 & 0.165 \\
\hline IDADE MET & -4.7 & 3.7 & -5.0 & -2.9 & 2.3 & -3.0 & 0.282 \\
\hline Visceral & -1.0 & 0.8 & -1.0 & -0.7 & 1.0 & 0.0 & 0.558 \\
\hline Braço Direito & -5.5 & 4.1 & -6.2 & -3.6 & 2.7 & -3.3 & 0.322 \\
\hline Braço Esq & 32.6 & 96.4 & -3.0 & -2.8 & 2.0 & -3.2 & 1.000 \\
\hline Antebraço D & -0.8 & 1.0 & -0.5 & -0.7 & 0.7 & -0.9 & 0.902 \\
\hline Antebraço E & 33.6 & 96.8 & -2.0 & -2.1 & 1.6 & -2.2 & 0.348 \\
\hline Peitoral & -3.2 & 2.6 & -4.0 & -0.9 & 2.6 & 0.0 & 0.124 \\
\hline Cintura & -3.9 & 4.4 & -3.0 & -3.5 & 3.8 & -3.5 & 0.848 \\
\hline Abdominal & -3.8 & 4.6 & -3.5 & -2.1 & 3.2 & -3.5 & 0.431 \\
\hline Quadril & -4.2 & 5.0 & -3.5 & -3.1 & 3.0 & -3.0 & 0.636 \\
\hline COXA D & -1.1 & 2.4 & -1.0 & 1.3 & 1.3 & 2.0 & 0.038 \\
\hline COXA E & -1.5 & 2.9 & -1.0 & 0.7 & 1.0 & 1.0 & 0.097 \\
\hline Panturrilha D & -0.3 & 0.6 & 0.0 & 0.1 & 0.7 & 0.0 & 0.304 \\
\hline Panturrilha E & -0.3 & 0.6 & 0.0 & 0.1 & 0.7 & 0.0 & 0.304 \\
\hline
\end{tabular}

Legenda: Antebraço D: antebraço direito, Antebraço E: antebraço esquerdo, Panturrilha D: panturrilha direita, Panturrilha E: panturrilha esquerda, Met basal: metabolismo basal, Idade Met: idade metabólica. ${ }^{*}$ IQ: Intervalo Interquartílico; ${ }^{* *}$ Média; ${ }^{* * *}$ Desvio-Padrão. ${ }^{a}$ Teste de Wilcoxon. ${ }^{b}$ Teste $t$ de Student pareado.

Fonte: Autores.

\section{Discussão}

A Síndrome dos ovários Policísticos apresenta vários desafios tanto para as mulheres que sofrem com a afecção como para o sistema de saúde e a sociedade. Os desafios se dão pela infertilidade e o risco de doenças relacionadas ao estilo de vida (obesidade, doenças metabólicas, cardiovasculares, oncológicas e psicológicas) e pelo fato de ainda não existir um tratamento integral que reverta ou cure os sintomas de SOP (Teede 2018).

A doença atinge mulheres principalmente no período fértil, sendo que nos estudos as amostras apresentam idade média de 30 anos de idade, concordando com os achados da tabela 01, que demonstrou que a maioria das participantes estavam na faixa dos 30 anos ou mais, porém, modificações no estilo de vida são recomendações de primeira linha de acordo com as diretrizes atuais (Lim, 2019; Ramos, 2016; Teede, 2018; Teede, 2018). Sousa et al. (2013), estudou o perfil metabólico de mulheres com SOP e no que se refere a amostra do estudo, a idade média foi de 26,3 anos, 65,4\% das mulheres eram solteiras, 
de cor parda (61,5\%), com ensino médio completo (52,6\%) e 79,5\% eram sedentárias. Esses dados diferem dos achados na tabela 01, onde a maioria das pacientes eram casadas, com ensino superior negra, se auto-intitularam negras e todas eram sedentárias.

Neste estudo foram comparados os resultados do treinamento concorrente (TC) e treinamento aeróbico (TA) em pacientes com SOP com sobrepeso ou obesas, no que tange parâmetros antropométricos, hormonais e metabólicos. Um dos problemas que podem ser causados pela SOP é o estado de hiperinsulinemia decorrente da resistência à insulina da (RI), fato este responsável pela elevação do androgênio no organismo. A insulina também age diminuindo a produção da proteína carregadora de hormônios sexuais (Steroid Hormone Binding Globuline - SHBG) no fígado e consequentemente esse processo resulta na elevação da concentração da testosterona livre na circulação sanguínea (Spritzer, 2014).

Outro fator que vem sendo relacionado a SOP é a obesidade. A relação entre a obesidade e os desequilíbrios hormonais em mulheres pode ser observada no estudo de Sales et al. (2015), onde foi relatado que os níveis de SHBG tendem a reduzir na medida em que a gordura corporal aumenta, causando aumento da fração livre de andrógenos no sangue, o que pode piorar um quadro de hiperandrogenimo. Os autores ainda afirmam que uma redução mínima de peso pode trazer melhorias significativas no quadro do paciente, como regularização do ciclo menstrual, atenuação das características hiperandrogênicas e possível melhora no quadro de infertilidade.

Bellver et al. (2018) também apoia a estreita relação da obesidade com o agravamento dos sintomas da SOP devido ao fato de uma das linhas de tratamento de SOP dar-se-á na alteração do estilo de vida, sendo recomendada a prática regular de atividade física e dietas balanceadas, com o objetivo ao início do tratamento de reduzir ao menos 5\% a $10 \%$ do peso da paciente. Esse dado sucinta ao objetivo deste estudo, que foi analisar os efeitos de duas modalidas de treinamento (TC e TA) realizado por 12 semanas.

O TC trata-se de um método que reúne exercícios aeróbicos e de força sendo executados na mesma sessão de treinamento. Essa combinação tem sido muito estudada para reduzir taxas de obesidade, devido ao alto gasto de energia durante e após o treinamento, fator este relacionado ao aumento do consumo de oxigênio após o exercício. Foi observado que os resultados do TC parecem ser mais eficazes que o TA para melhorar o perfil lipídico e medidas antropométricas (Inoue et al, 2015).

Já Monteiro et al., (2015), demonstrou que houve diminuição significativa do percentual de gordura corporal total no TA e no TC em comparação com o grupo controle (GC), concluindo que ambos os treinamentos podem trazer benefícios para os perfis metabólicos associados à obesidade abdominal. Medeiros et al. (2015), avaliou o efeito do TC sobre o peso e IMC de mulheres obesas submetidas a cinco sessões e três sessões semanais em intensidade moderada. Os autores afirmaram que ambas as frequências reduziram o peso corporal e o IMC, porém, o TC aplicado cinco sessões semanais mostrou redução do percentual de gordura e aumento da taxa metabólica basal. Esses estudos apoiam os resultados da tabela 02, que demonstrou que um programa de TC foi capaz de reduzir o peso e o IMC das participantes.

Além do TC, esta pesquisa também investigou os efeitos do TA em mulheres com SOP com sobrepeso ou obesidade. O exercício aeróbico é uma modalidade altamente recomendada para pessoas obesas devido aos benefícios sobre a composição corporal, o perfil lipídico, pressão arterial, controle glicêmico e aptidão cardiorrespiratória (Medeiros et al., 2015).

Corroborando com os achados das tabelas 03, Ribeiro et al. (2021), realizou um estudo controlado randomizado onde avaliou os efeitos do TAC (treinamento aeróbico contínuo) (n=28), TAI (treinamento aeróbico intermitente)(n=29) e o GC (sem treinamento; $\mathrm{n}=30$ ) em mulheres com SOP. As avaliações ocorreram no início e após 16 semanas de intervenção. Após a intervenção, foi encontrada redução significativa da circunferência da cintura $(C C)$ nos grupos TAC $(p=0,045)$ e TAI $(p=$ 0,049). Por outro lado, um aumento significativo na CC foi encontrado no grupo GC ( $\mathrm{p}=0,014)$. A circunferência do quadril (CQ) foi significativamente reduzida no grupo TAC $(\mathrm{p}=0,032)$. Além disso, houve redução significativa da RCQ (relação 
cintura-quadril) no grupo TAI $(\mathrm{p}=0,012)$.

Outro autor que demonstrou que o exercício pode modificar diversos parâmetros em mulheres com SOP foi Benham et al. (2018), que desenvolveu uma revisão sistemática com metanálise onde buscou determinar o impacto do treinamento físico em mulheres com SOP. O exercício aeróbico foi avaliado em seis estudos, o treinamento de resistência foi avaliado em um estudo, quatro estudos avaliaram uma combinação de aeróbio e resistência treinamento e três estudos analisaram o impacto de vários tipos de atividade física que não foram especificados. Foi observado que os exercícios podem melhorar a regularidade menstrual, gravidez e taxas de ovulação. Na metanálise descobriu-se que o exercício melhorou o perfil lipídico e diminuiu a circunferência da cintura, a pressão arterial sistólica e a insulina em jejum. Esses dados estudos entram em sintonia com a tabela 04 e 05, que demonstra que TC e TA conseguiram reduzir taxas metabólicas e hormonais. Ainda de acordo com esses achados, o estudo de Ribeiro et al. (2021), demonstrou que os níveis de testosterona nos grupos TAC (p <0,001) e TAI (p $=0,019$ ) foram significativamente reduzidos, estando de acordo com a tabela 05.

A tabela 06 mostra a comparação entre o TA e TC, sendo os grupos foram homogeneos, com redução significativa apenas na coxa D. Como já explicado no estudo, a literatura pesquisada carece de estudos que comparem os TC e TA em pacientes com SOP, porém, estudos demonstram que os tratamentos vem apresentando resultados significativos. O estudo de Cruz (2017), que investigou os efeitos do TC em mulheres obesas com síndrome metabólica demonstrou que o TC aplicado ao grupo experimental conseguiu reduzir peso $(\mathrm{p}=0,000)$, IMC $(\mathrm{p}=0,000)$, cintura $(0,005)$, gordura $(\mathrm{p}=0,000)$, braço $\mathrm{D}$ e $\mathrm{E}$ ( $\mathrm{p}=0,000)$ e colesterol LDL ( $\mathrm{p}=0,000)$, já o grupo controle não obteve resultados significativos nesses parâmetros. Esses dados concordam com os resultados das tabelas 02 e 04 que mostram resultados similares no grupo TC. O TA também mostrou resultados favoráveis em vários parâmetros como redução do IMC, do peso, relação cintura-quadril, circunferência da cintura, gordura, colesterol HDL e LDL e triglicerídeos em mulheres com SOP (Abazar et al, 2015; Costa et al, 2018).

Tendo em vista a profunda revisão de pesquisas relacionadas ao tema nas bases de dados, parece que este é o primeiro estudo a comparar os efeitos de um protocolo de TA e TC na composição corporal, parâmetros metabólicos e endócrinos em mulheres com SOP obesas e com sobrepeso. Este fator torna relevante a realização desta pesquisa para melhorar o atendimento a essa população, além de fornecer mais opções de tratamento a mesmas.

Contudo, apesar da indicação constante de alterações no estilo de vida para melhor tratamento da SOP, incluindo a realização de exercícios físicos, como já citado no decorrer deste trabalho, a literatura conta com escasso material sobre o tema, principalmente sobre TC, não dispondo de outro trabalho que compare o TC com o TA. Os estudos envolvendo treinamento aeróbico encontram-se em maior quantidade, porém ainda há um grande número de estudos piloto, revisões ou estudos que analisam outros parâmetros, fugindo a analise bioquímica e antropométrica, focando mais na qualidade de vida, níveis de força e outros parâmetros dificultando a discussão dos achados nesta pesquisa com o que as publicações disponíveis. Para minimizar os efeitos dessa limitação, foi feita uma pesquisa ampla em várias bases de dados, como Pubmed, SciELO, Biblioteca Virtual em Saúde, e em vários idiomas, como inglês, português e espanhol, visando explorar profundamente o que há na literatura.

\section{Conclusão}

Os resultados do estudo permitem concluir que:

- O treinamento físico concorrente reduz as medidas antropométricas de mulheres obesas ou com sobrepeso portadora da SOP após 12 semanas de treino, principalmenteo IMC, gordura corporal, idade metabólica e massa muscular;

- O treinamento físico aeróbico reduz as medidas antropométricas de mulheres obesas ou com sobrepeso portadora da SOP após 12 semanas de treino, principalmente o IMC, gordura corporal, idade metabólica, 
metabolismo basal,circunferências: abdominal, peitoral e de quadril;

- Os treinamentos físicos concorrente e aeróbico foram eficazes na melhora dos parâmetros metabólicos e hormonais, sendo mais evidente no TOTG-75 (2h) e colesterol total para os dois treinos; HDL, LDL e glicemia para oaeróbico e triglicerídeos para o concorrente.

- Os dois treinamentos possuem eficácias semelhantes em reduzir as medidas antropométricas após 12 semanas de treinamento (três vezes por semana), como forma de tratamento de mulheres obesas ou com sobrepeso portadorasda síndrome dos ovários policísticos.

\section{Considerações Gerais}

Os tratamentos envolvendo a SOP ainda são em sua maioriamedicamentosos, porém tratamentos conservadores como mudanças no estilo de vida podem ser bons aliados ao tratamento, trazendo maiores benefícios a esse público. A realização deste estudo busca, além de analisar modalidades de treinamentos físicos em vários parâmetros, incentiva a realização de mais estudos que investiguem os efeitos dos exercícios físicos em mulheres com SOP e outros distúrbios de cunho hormonal.

Nos resultados foi possível verificar reduções significativas em vários parâmetros como peso, IMC, gordura e nos exames laboratoriais e metabólicos. A falta de eventos adversos durante a execução da pesquisa demonstra que ambas as modalidades são seguras e podem ser indicadas a mulheres com SOP. Na comparação entre os treinamentos ainda foi visto um comportamento similar, com maioria dos parâmetros com índice de significância menor que 5\%, o que demonstra que ambos os treinamentos foram eficazes ao público aplicado e sugere que ambos podem ser utilizados como tratamento complementar a pessoas com distúrbioshormonais.

Apesar das limitações, os dados encontrados neste estudo fornecem uma base sobre o tema, abrindo caminho para a formulação de novas pesquisas científicos na área. Obteve-se ao final do programa o resultado de quatro mulheres grávidas, demonstrando que a atividade física voltada para o emagrecimento favorece essa população. Para estudos futuros, sugere-se que sejam avaliados outros fatores como qualidade de vida, percepção pessoal quanto aos resultados obtidos pelas participantes e correlação dos resultados aos tratamentos medicamentoso sem uso pelas participantes.

Ademais, a escassez de artigos na literatura no que se relaciona ao tema demonstra a necessidade de mais pesquisas na área, especialmente comtratamentos coadjuvantes baseados em atividade física e mudanças alimentares.

\section{Referências}

Abazar, E., Taghian, F., Mardanian, F., \& Forozandeh, D. (2015). Effects of aerobic exercise on plasma lipoproteins in overweight and obese women with polycystic ovary syndrome. Advanced biomedical research, 4

Abeso. Diretrizes Brasileiras de Obesidade; 2016. (4a ed.), 15-22.

American College Of Sports Medicine (ACSM). Diretrizes do ACSM para os testes de esforço e sua prescrição. 2007. (7a ed.), GuanabaraKoogan.

Azziz, R., Carmina, E., Dewailly, D., Diamanti-Kandarakis, E., Escobar-Morreale, H. F., Futterweit, W., \& Witchel, S. F. (2006). Criteria for defining polycystic ovary syndrome as a predominantly hyperandrogenic syndrome: an androgen excess society guideline. The Journal of Clinical Endocrinology \& Metabolism, 91(11), 4237-4245.

Baranova, A., Tran, T. P., Birerdinc, A., \& Younossi, Z. M. (2011). Systematic review: association of polycystic ovary syndrome with metabolic syndrome and non-alcoholic fatty liver disease. Alimentary pharmacology \& therapeutics, 33(7), 801-814.

Barber, T. M., McCarthy, M. I., Franks, S., \& Wass, J. A. (2007). Metabolic syndrome in polycystic ovary syndrome. Endokrynologia Polska, 58(1), 34-41.

Barber, Thomas M. et al. (2006). Obesity and polycystic ovary syndrome. ClinicalEndocrinology, 65(2), 137-145.

Barber, T. M., Dimitriadis, G. K., Andreou, A., \& Franks, S. (2016). Polycystic ovary syndrome: insight into pathogenesis and a common association with insulin resistance. Clinical Medicine, 16(3), 262.

Barnard, L., Ferriday, D., Guenther, N., Strauss, B., Balen, A. H., \& Dye, L. (2007). Quality of life and psychological well being in polycystic ovary syndrome. Human reproduction, 22(8), 2279-2286. 
Bellver, J., Rodríguez-Tabernero, L., Robles, A., Muñoz, E., Martínez, F., Landeras, J., ... \& Acevedo, B. (2018). Polycystic ovary syndrome throughout a woman's life. Journal of assisted reproduction and genetics, 35(1), 25-39.

Benham, J. L., Yamamoto, J. M., Friedenreich, C. M., Rabi, D. M., \& Sigal, R. J. (2018). Role of exercise training in polycystic ovary syndrome: a systematic review and meta-analysis. Clinical obesity, 8(4), 275-284.

Borg, G. A. (1982). Psychophysical bases of perceived exertion. Medicine \& science in sports \& exercise

Brasil. (2012). Ministério da Saúde. Resoluçãon 466/12. Conselho Nacional de Pesquisa com Seres Humanos. Diário Oficial da União. Brasília.

Bulsara, J. P., Patel, P., Soni, A., \& Acharya, S. (2021). A review on brief insight into Polycystic Ovarian syndrome. Endocrine and Metabolic Science, 100085 .

Carmina, E., \& Azziz, R. (2006). Diagnosis, phenotype, and prevalence of polycystic ovary syndrome. Fertility and sterility, 86, S7-S8.

Costa, E. C., Sá, J. C. F. D., Stepto, N. K., Costa, I. B. B., Farias Junior, L. F., Moreira, S. D. N. T., \& Azevedo, G. D. D. (2018). Aerobic training improves quality of life in women with polycystic ovary syndrome.

Costa, R. F. (1999). Qual a melhor técnica de avaliação da composição corporal. Revista Nutrição em Pauta. São Paulo, 7(37), 31-35.

Cruz, I. D. S. D. (2017). Síndrome metabólica e condicionamento físico parciais de mulheres jovens obesas submetidas a um protocolo de treinamento concorrente.Tese de doutorado [Internet].

Delitala, A. P., Capobianco, G., Delitala, G., Cherchi, P. L., \& Dessole, S. (2017). Polycystic ovary syndrome, adipose tissue and metabolic syndrome. Archives of gynecology and obstetrics, 296(3), 405-419.

Ding, T.et al. The prevalence of polycystic ovary syndrome in reproductive-aged women of different ethnicity: a systematic review and meta-analysis. Onco Target, 8(56), 96351-96358.

Drosdzol, A., Skrzypulec, V., Mazur, B., \& Pawlińska-Chmara, R. (2007). Quality of life and marital sexual satisfaction in women with polycystic ovary syndrome. Folia histochemica et cytobiologica, 45(I), 93-97.

Ehrmann, D. A. (2005). Polycystic ovary syndrome. New England Journal of Medicine, 352(12), 1223-1236.

Forslund, M., Landin-Wilhelmsen, K., Schmidt, J., Brännström, M., Trimpou, P., \& Dahlgren, E. (2019). Higher menopausal age but no differences in parity in women with polycystic ovary syndrome compared with controls. Acta obstetricia et gynecologica Scandinavica, 98(3), 320-326.

Hudecova, M., Holte, J., Olovsson, M., Larsson, A., Berne, C., \& Sundstrom-Poromaa, I. (2011). Prevalence of the metabolic syndrome in women with a previous diagnosis of polycystic ovary syndrome: long-term follow-up. Fertility and sterility, 96(5), 1271-1274.

HUTCHISON, Samantha K. et al. Effects of exercise on insulin resistance and body composition in overweight and obese women with and without polycystic ovary syndrome. The Journal of Clinical Endocrinology \& Metabolism, 96(1), E48-E56.

Inoue, D. S., De Mello, M. T., Foschini, D., Lira, F. S., Ganen, A. D. P., Campos, R. M. D. S., \& Dâmaso, A. R. (2015). Linear and undulating periodized strength plus aerobic training promote similar benefits and lead to improvement of insulin resistance on obese adolescents. Journal of Diabetes and its Complications, 29(2), 258-264.

Kandaraki, E., Christakou, C., \& Diamanti-Kandarakis, E. (2009). Metabolic syndrome and polycystic ovary syndrome... and vice versa. Arquivos Brasileiros de Endocrinologia \& Metabologia, 53, 227-237.

Kim, J. J., \& Choi, Y. M. (2013). Dyslipidemia in women with polycystic ovary syndrome. Obstetrics \& gynecology science, 56(3), 137-142.

Kogure, G. S., Silva, R. C., Picchi Ramos, F. K., Miranda-Furtado, C. L., Lara, L. A. D. S., Ferriani, R. A., \& Dos Reis, R. M. (2015). Women with polycystic ovary syndrome have greater muscle strength irrespective of body composition. Gynecological Endocrinology, 31(3), 237-242.

Legro, R. S. (2000). The genetics of obesity Lessons for polycystic ovary syndrome. Annals of the New York Academy of Sciences, 900(1), 193-202.

Lim, S. S., Hutchison, S. K., Van Ryswyk, E., Norman, R. J., Teede, H. J., \& Moran, L. J. (2019). Lifestyle changes in women with polycystic ovary syndrome. Cochrane Database of Systematic Reviews, (3)..

Lim, S. S., Davies, M. J., Norman, R. J., \& Moran, L. J. (2012). Overweight, obesity and central obesity in women with polycystic ovary syndrome: a systematic review and meta-analysis. Human reproduction update, 18(6), 618-637.

Materko, W., Neves, C. E. B., \& Santos, E. L. (2007). Modelo de predição de uma repetição máxima (1RM) baseado nas características antropométricas de homens e mulheres. Revista Brasileira de Medicina do Esporte, 13, 27-32.

Medeiros, N. D. S., de Abreu, F. G., Colato, A. S., de Lemos, L. S., Ramis, T. R., Dorneles, G. P., \& Dani, C. (2015). Effects of concurrent training on oxidative stress and insulin resistance in obese individuals. Oxidative medicine and cellular longevity, 2015.

Monteiro, P. A., Chen, K. Y., Lira, F. S., Saraiva, B. T. C., Antunes, B. M. M., Campos, E. Z., \& Freitas, I. F. (2015). Concurrent and aerobic exercise training promote similar benefits in body composition and metabolic profiles in obese adolescents. Lipids in health and disease, 14(1), 1-9.

Ramos, F. K. P., Lara, L. A. D. S., Kogure, G. S., Silva, R. C., Ferriani, R. A., Silva de Sá, M. F., \& Reis, R. M. D. (2016). Quality of life in women with polycystic ovary syndrome after a program of resistance exercise training. Revista Brasileira de Ginecologia e Obstetrícia, 38, 340-347.

Rezende, F., Rosado, L., Franceschinni, S., Rosado, G., Ribeiro, R., \& Bouzas Marins, J. C. (2007). Revisão crítica dos métodos disponíveis para avaliar a composição corporal em grandes estudos populacionais e clínicos. Archivos Latinoamericanos de nutricion, 57(4), 327-334. 
Ribeiro, V. B., Kogure, G. S., Lopes, I. P., Silva, R. C., Pedroso, D. C. C., Ferriani, R. A., \& Reis, R. M. D. (2019). Association of measures of central fat accumulation indices with body fat distribution and metabolic, hormonal, and inflammatory parameters in women with polycystic ovary syndrome. Archives of endocrinology and metabolism, 63, 417-426.

Ribeiro, V. B., Lopes, I. P., Dos Reis, R. M., Silva, R. C., Mendes, M. C., Melo, A. S., \& Lara, L. A. D. S. (2021). Continuous versus intermittent aerobic exercise in the improvement of quality of life for women with polycystic ovary syndrome: A randomized controlled trial. Journal of health psychology, 26(9), 1307-1317.

Rossi, F. E. (2016). Comportamento da prática habitual de atividade física, ingestão alimentar, composição corporal e perfil metabólico em mulheres pósmenopausa, após programas de treinamentos concorrente e aeróbio. Universidade Estadual Paulista, Instituto de Biociências de Rio Claro, 63 f.

Eshre, T. R., \& ASRM-Sponsored PCOS Consensus Workshop Group. (2004). Revised 2003 consensus on diagnostic criteria and long-term health risks related to polycystic ovary syndrome. Fertility and sterility, 81(1), 19-25.

Sales, M. F., Sóter, M. O., Cândido, A. L., Reis, F. M. D., Sousa, M. O., \& Fernande, A. P. (2015). Ferriman-Gallwey Score correlates with obesity and insulin level in Polycystic Ovary Syndrome-an observational study. Rev Soc Bras Clin Med, 13(2), 107-110.

Sattar, N. (2011). Polycystic Ovary Syndrome. In: BYRNE, C.; WILD, S. The MetabolicSyndrome. Blackwell Publishing Ltd., p. $278-298$.

Sattar, N. (2006). Vascular and metabolic issues in PCOS. Women's vascular health. Boca Raton: Taylor \& Francies Group, $265-79$.

Setji, T. L., \& Brown, A. J. (2014). Polycystic ovary syndrome: update on diagnosis and treatment. The American journal of medicine, 127(10), 912-919.

Sharma, K., \& Srivastava, S. K. (2020). The Role of Life style Modification in Management of Polycystic Ovary Syndrome. Indian Journal of Public Health, 11(11), 107.

Sidhwani, S., Scoccia, B., Sunghay, S., Stephens-Archer, C. N., Mazzone, T., \& Sam, S. (2011). Polycystic ovary syndrome is associated with atherogenic changes in lipoprotein particle number and size independent of body weight. Clinical endocrinology, 75(1), 76-82.

Sigal, R. J., Kenny, G. P., Boulé, N. G., Wells, G. A., Prud'homme, D., Fortier, M., \& Jaffey, J. (2007). Effects of aerobic training, resistance training, or both on glycemic control in type 2 diabetes: a randomized trial. Annals of internal medicine, 147(6), 357-369.

Sousa, R. M. L. D., Chein, M. B. D. C., Silva, D. S. M. D., Dutra, M. B., Navarro, P. A. D. A. S., Figueiredo Neto, J. A. D., \& Brito, L. M. O. (2013). Perfil metabólico em mulheres de diferentes índices de massa corporal com síndrome dos ovários policísticos. Revista Brasileira de Ginecologia e Obstetrícia, 35, 413-420.

Spritzer, P. M. (2014). Polycystic ovary syndrome: reviewing diagnosis and management of metabolic disturbances. Arquivos Brasileiros de Endocrinologia \& Metabologia, 58, 182-187.

Tasali, E., Van Cauter, E., \& Ehrmann, D. A. (2008). Polycystic ovary syndrome and obstructive sleep apnea. Sleep medicine clinics, 3(1), $37-46$.

Teede, H. J., Misso, M. L., Costello, M. F., Dokras, A., Laven, J., Moran, L., \& Norman, R. J. (2018). Recommendations from the international evidencebased guideline for the assessment and management of polycystic ovary syndrome. Human reproduction, 33(9), $1602-1618$.

Teede, H. J., Joham, A. E., Paul, E., Moran, L. J., Loxton, D., Jolley, D., \& Lombard, C. (2013). Longitudinal weight gain in women identified with polycystic ovary syndrome: results of an observational study in young women. Obesity, 21(8), 1526-1532.

Teede, H. J., Misso, M. L., Costello, M. F., Dokras, A., Laven, J., Moran, L., \& Norman, R. J. (2018). Recommendations from the international evidencebased guideline for the assessment and management of polycystic ovary syndrome. Human reproduction, 33(9), 1602-1618

Teede, H., Deeks, A., \& Moran, L. (2010). Polycystic ovary syndrome: a complex condition with psychological, reproductive and metabolic manifestations that impacts on health across the lifespan. BMC medicine, 8(1), 1-10.

Turan, V., Mutlu, E. K., Solmaz, U., Ekin, A., Tosun, O., Tosun, G., ... \& Malkoc, M. (2015). Benefits of short-term structured exercise in non-overweight women with polycystic ovary syndrome: a prospective randomized controlled study. Journal of physical therapy science, $27(7), 2293-2297$.

Wanderley, F. A., Moreira, A., Sokhatska, O., Palmares, C., Moreira, P., Sandercock, G.,\& Carvalho, J. (2013). Differential responses of adiposity, inflammation and autonomic function to aerobic versus resistance training in older adults. Experimental gerontology, 48(3), 326-333.

Wild, S., Pierpoint, T., McKeigue, P., \& Jacobs, H. (2000). Cardiovascular disease in women with polycystic ovary syndrome at long-term follow-up: a retrospective cohort study. Clinical endocrinology, 52(5), 595-600. 